\title{
Caracterización de armas antiguas. Las inclusiones testigos de calidad, proceso y origen
}

\author{
G. Rosado* y J.C. García*
}

\begin{abstract}
Resumen El trabajo que se presenta constituye una muestra de las posibilidades en la aplicación de las modernas técnicas de caracterización a los materiales arqueológicos: desde armas hasta objetos de uso común. En concreto, se presentan los datos obtenidos en armas de diferentes épocas, desde una falcata ibérica hasta una espada de Mondragón y un yunque más moderno. Se inicia el camino para realizar un análisis de procedencia al poderse relacionar los componentes del material base, mediante las inclusiones presentes, con los minerales de origen y los escoriales donde se han procesado.
\end{abstract}

Palabras clave Inclusiones. Arqueometalurgia.

\section{Characterisation of ancient weapons. Inclusions as witnesses of quality, process and origin}

\begin{abstract}
This work shows the possibility of using new techniques to characterise archaeological items, from weapons to common objects. To sum up, data from weapons manufactured in different ages are shown, from an Iberian "falcata", to a Mondragón sword and also a more actual anvil. Preliminary steps are carried out to start an analysis of base material origin according to existing inclusions and to relate this with origin mineral and slag dumps where they were processed.
\end{abstract}

Keywords Inclusions. Archaeometallurgy.

\section{INTRODUCCIÓN}

La caracterización de objetos arqueológicos es una actividad que se está demandando cada vez con mayor frecuencia; bien sea el caso de útiles concretos como espadas, monedas, herramientas y aperos agrícolas, residuos de procesos metalúrgicos como escorias y masas metálicas; o bien, materiales en el amplio sentido de la palabra, procedentes de yacimientos arqueológicos tales como el de Santa Criz en Navarra o el de La Serreta de Alcoy donde se ha dispuesto para el análisis del material de casi noventa tumbas.

Las cuestiones a las que se ha intentado dar respuesta, aparte de la propia caracterización de los objetos, son de lo más curioso: el Museo de Navarra se preguntaba sobre la autenticidad de monedas medievales y procedimientos de la época para la falsificación de las mismas. También, para el museo de Navarra se han aportado datos sobre los procesos metalúrgicos desarrollados en el poblado romano de Santa Criz. La incógnita a resolver, en el caso del yacimiento ibérico de La Serreta, era el efecto del rito funerario de cremación en las armas de la época. El amplio estudio realizado, ha permitido distinguir qué armas habían pasado realmente por la pira funeraria y como habían sido afectadas por la temperatura; el destino bélico o ceremonial, el proceso de fabricación y hasta la calidad de las mismas.

En este momento, se colabora en los estudios de la cuenca metalúrgica de Legazpia y en la confirmación científica de la relación entre las famosas espadas toledanas y el no menos citado históricamente acero de Mondragón ${ }^{[1]}$.

Esta amplia actividad ha permitido establecer un protocolo de análisis que facilita la obtención de un máximo de información con un sacrificio patrimonial o estético inapreciable. A tal efecto, se han aplicado de forma novedosa ensayos clásicos como la inspección visual y la réplica metalográfica de la estructura, se han puesto a punto métodos

(*) FUNDACIÓN INASMET. Po Mikeletegi, 2; 20009 San Sebastián 
para la extracción de muestras como es la electroerosión por hilo y se han aplicado varias de las herramientas de la panoplia analítica como el microscopio electrónico con microsonda acoplada y la aceleración de partículas.

En esta ocasión, el trabajo se refiere en exclusiva, a la información obtenida mediante el análisis de las inclusiones descubiertas en objetos metálicos de carácter siderúrgico, es decir, armas y herramientas de hierro y acero.

\section{MATERIALES Y PROCEDIMIENTO EXPERIMEN- TAL}

Los datos analíticos que se presentan corresponden a las piezas siguientes:

- Falcata ibérica, fechada alrededor del siglo III antes de Cristo.

- Espada realizada con acero de Mondragón (siglos XVI/XVII).

- Yunque para moletear hoces recuperado en un caserío de Elorrio (siglos XVIII/XIX).

Una vez seleccionada una zona óptima para el estudio, las muestras son extraídas y, en este caso, analizadas mediante inspección visual, análisis metalográfico y análisis mediante microsonda EDS acoplada a un microscopio electrónico de barrido.

El estudio se ha planteado siguiendo básicamente el proceso tradicional de elaboración de los materiales siderúrgicos tal como lo describen autores ya clásicos de la arqueometalurgia como Tylecotte, Claude Domerque o Serneels ${ }^{[2-5]}$.

\section{RESULTADOS Y DISCUSIÓN}

\subsection{La reducción}

Como es sabido, en Occidente no se consigue licuar el hierro hasta bien avanzada la Edad Media gracias al empleo de la fuerza hidráulica para mover los barquines o alimentar las trompas. Según esto, en un principio, el hierro se obtenía por reducción del mineral en hornos bajos no pasando la masa del estado pastoso. Una de las consecuencias era la presencia de partículas de escoria en la masa del metal que se intentaba paliar por sucesivas operaciones de refino o "amasado" en el yunque.

Las aludidas inclusiones gozan de características peculiares que es posible identificar con los medios analíticos actuales. Vistas al microscopio, presentan un aspecto granuloso propio del conglomerado de óxidos y silicatos que las configuran (ver cadena superior, figuras 1 y 2 correspondientes a la falcata ibérica). Si se aplica la microsonda se verá que en la composición (ver línea A en tabla I) aparece el potasio en cantidad suficiente como para poder interpretar que nos hallamos ante un proceso de reducción en el que se ha utilizado el carbón vegetal como combustible. En las cenizas de este carbón se halla el potasio en cantidades importantes y tiene la propiedad de incorporarse a la escoria durante el proceso de reducción.

Parece de interés añadir aquí que, además, la presencia del potasio tiene la propiedad inestimable de bajar el nivel de temperatura necesaria para comenzar la formación de escoria líquida y por tanto el punto crítico de comienzo de la movilidad entre fases sólidas, semisólidas y líquidas que permiten la reducción. Si se comparan los gráficos ternarios (Figs. 3 y 4), se verá que el diferencial de temperatura atribuible a la presencia del potasio

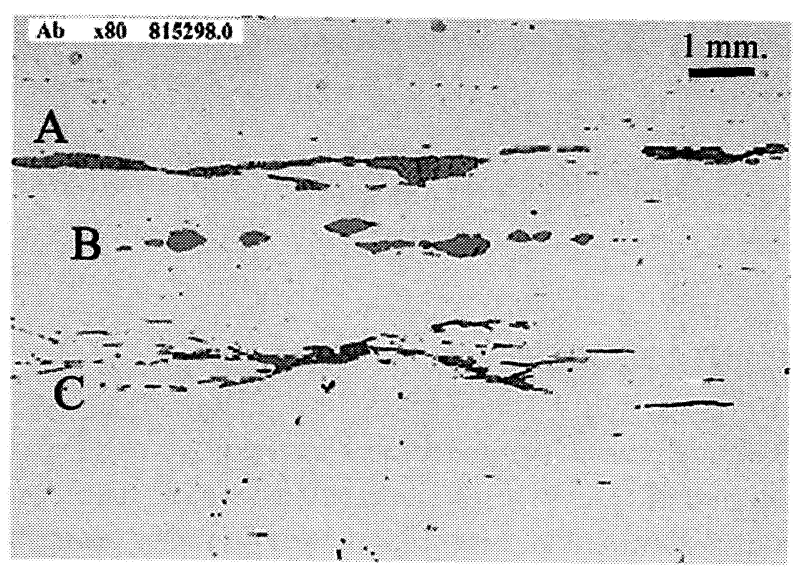

Figura 1. Inclusiones en falcata.

Figure 1. Inclusions in "falcata".

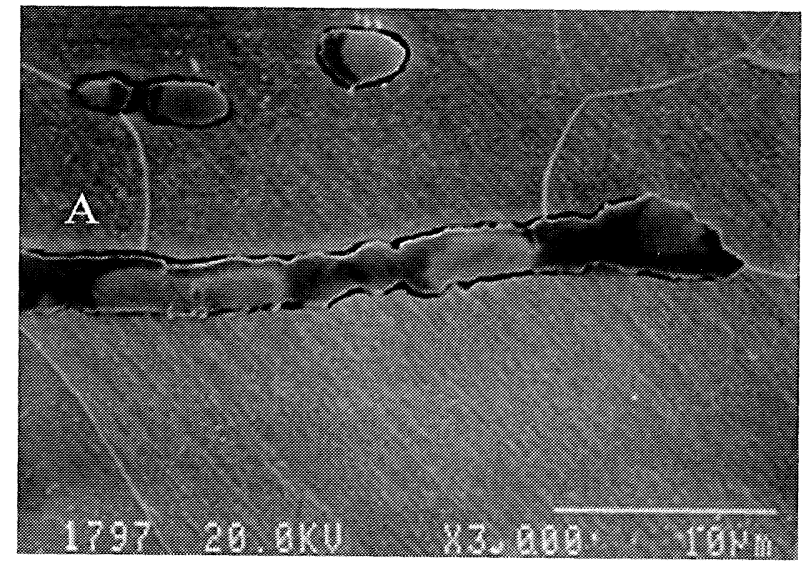

Figura 2. Inclusiones en falcata (MEB).

Figure 2. Inclusions in "falcata" (SEM). Rev. Metal. Madrid Vol. Extr. (2005) 101-106 
Tabla I. Análisis de inclusiones

Table I. Analysis of inclusions

\begin{tabular}{llccccccccc}
\hline Ref. & \multicolumn{1}{c}{ Pieza } & Fig. & $\mathrm{Fe}_{2} \mathrm{O}_{3}$ & $\mathrm{SiO}_{2}$ & $\mathrm{Al}_{2} \mathrm{O}_{3}$ & $\mathrm{~K}_{2} \mathrm{O}$ & $\mathrm{SO}_{2}$ & $\mathrm{CaO}$ & $\mathrm{MnO}_{2}$ & $\mathrm{Tipo}^{2}$ \\
\hline $\mathrm{A}$ & Falcata & $1 \mathrm{y} 2$ & 51 & 29 & 5 & 3 & - & 10 & 0,6 & Reducción \\
$\mathrm{B}$ & Falcata & 1 & 99 & - & - & - & - & - & 0,6 & Mineral \\
$\mathrm{C}$ & Falcata & 1 & 13 & 82 & 5 & - & - & - & - & Arena \\
$\mathrm{D}$ & Falcata & 7 & 99 & - & - & - & - & - & - & Cascarilla \\
$\mathrm{E}$ & Esp. Mond. & 8 & 50 & 5 & 25 & 4 & - & 7 & 9 & Reducción \\
$\mathrm{F}$ & Esp. Mond. & 8 & 92 & 7 & - & - & - & - & - & Cascarilla \\
$\mathrm{G}$ & Yunque & 5 & 54 & 33 & - & - & 2 & - & 10 & Reducción \\
$\mathrm{H}$ & Yunque & 5 & 45 & - & 54 & - & - & - & - & Pared \\
\hline
\end{tabular}

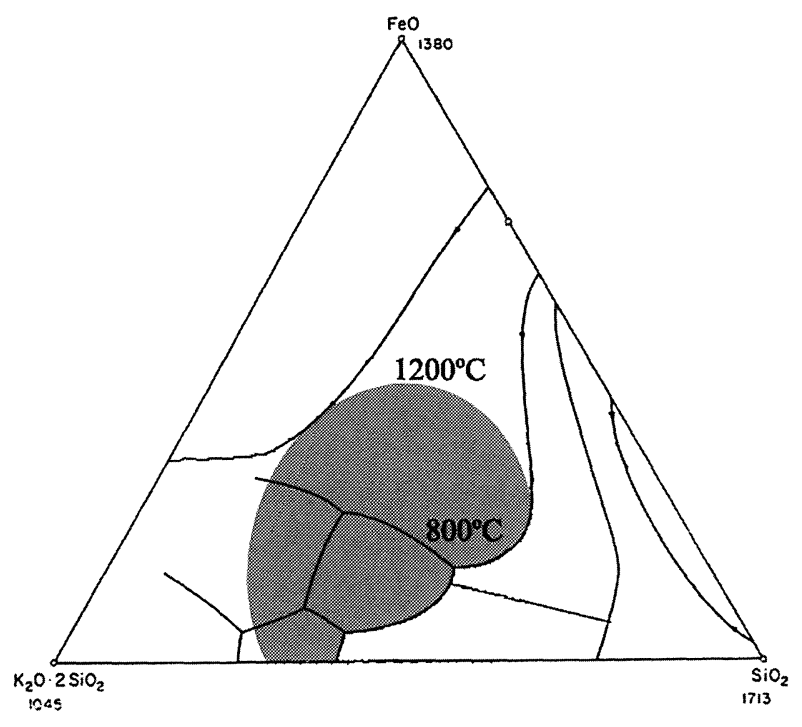

Figura 3. Sistema ternario $\mathrm{K}_{2} \mathrm{O}-\mathrm{FeO}-\mathrm{SiO}_{2}$.

Figure 3. $\mathrm{K}_{2} \mathrm{O}-\mathrm{FeO}-\mathrm{SiO}_{2}$ ternary diagram.

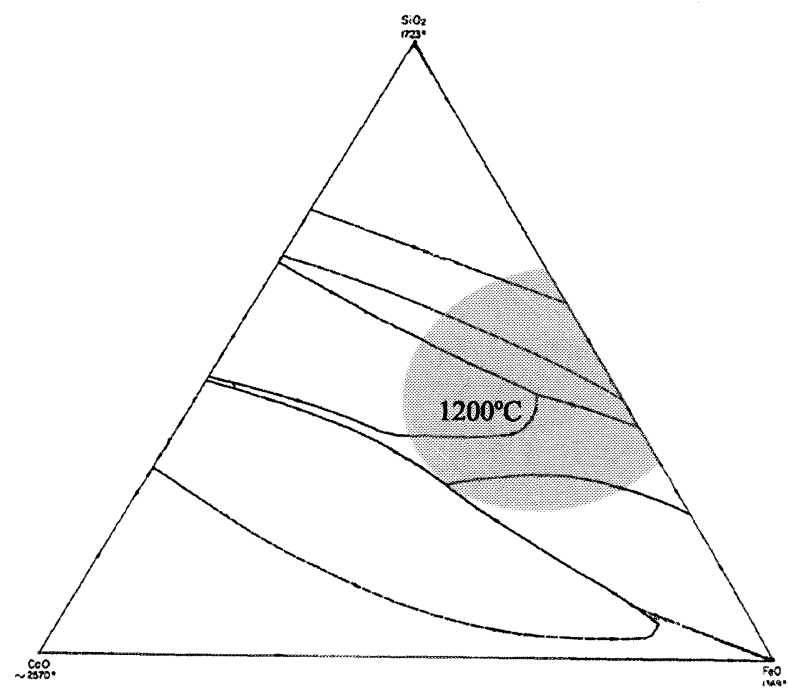

Figura 4. Sistema ternario $\mathrm{FeO}-\mathrm{CaO}-\mathrm{SiO}_{2}$.

Figure 4. $\mathrm{FeO}-\mathrm{CaO}-\mathrm{SiO}_{2}$ ternary Diagram. permite situarse en un campo eutéctico, más de acuerdo con los medios tecnológicos de la época que cuando este elemento se encuentra ausente. Es decir, la reducción era "posible" a partir de los 800 ${ }^{\circ} \mathrm{C}$ de temperatura, asequibles a un horno bajo sin apoyo de ventilación forzada.

Siguiendo con la composición aludida más arriba, se observa un contenido en calcio ciertamente relevante pero se puede atribuir a una importante aportación del entorno próximo (en el carbón, el mismo mineral o la pared del horno) más que a una adición inteligente para incrementar el rendimiento en hierro del proceso en base a sustituir parte del óxido de hierro necesario para formar la escoria por óxido de calcio. Por último, el contenido en manganeso es una primera referencia al mineral de origen.

La figura 5 muestra micrografías del yunque que, al parecer, ha sido confeccionado "calzando" un soporte de hierro con un inserto de acero, (Fig. 6). La micrografía superior muestra, a pocos aumentos, la zona de soldadura de ambos metales,

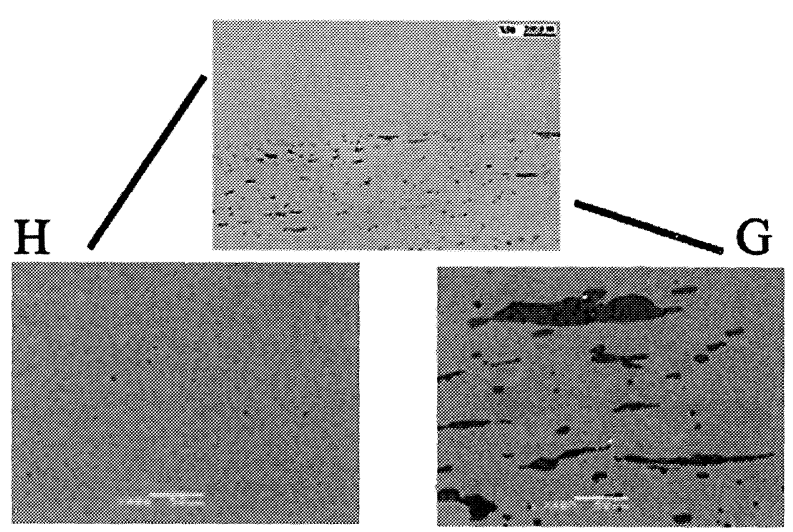

Figura 5. Inclusiones en yunque.

Figure 5. Inclusions in anvil. 

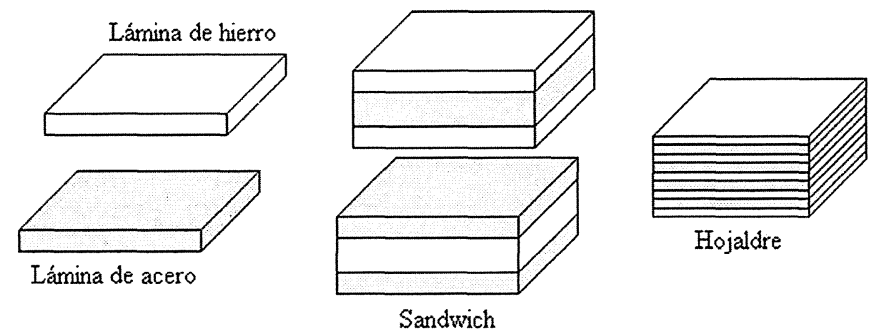

Hojaldre
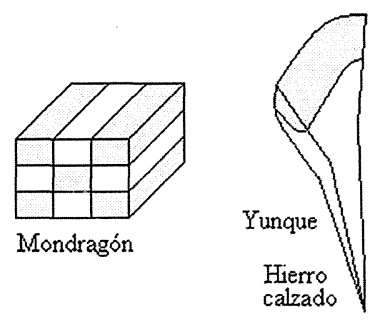

Figura 6. Esquema de fabricación de herramientas.

Figure 6. Scheme of tools' fabrication.

la inferior derecha, correspondiente al hierro, presenta una enorme cantidad de inclusiones de gran tamaño y del tipo de reducción. El análisis de estas últimas (ver análisis F en tabla I) contiene azufre en vez de potasio lo que puede indicar que corresponde a un proceso de reducción en el que se ha utilizado el carbón de piedra como combustible, lo cual puede ser verosímil a partir de finales del siglo XVIII.

\subsection{La compactación y el refino}

Las siguientes operaciones a la reducción son la compactación y el refino de la esponja de hierro obtenida que tenía por objeto la eliminación de una parte de las escorias retenidas en la masa metálica. Estas operaciones se realizaban en el suelo o en una mesa de piedra próxima al horno.

No es corriente encontrar materiales ocluidos externos a la propia operación de reducción pero, en el caso de la cadena intermedia de las tres que se presentan en la figura 1 , no hay mucho margen a la interpretación ya que su análisis por la microsonda (ver análisis B de la tabla I) muestra que están constituidas casi exclusivamente por óxido de hierro, estando ausentes los silicatos propios de la escoria y el potasio procedente de la reducción. Solamente se detecta la presencia de manganeso en proporción similar a la comentada más arriba, lo que lleva a concluir que se está ante una porción del mineral de origen que se ha incorporado a la masa metálica en la compactación o el amasado para el refino, realizado probablemente en el mismo lugar donde se fragmentaba el mineral.

\subsection{La forja de armas y herramientas}

Es más frecuente la presencia de vestigios de esta etapa que son de los tipos siguientes:
- Inclusiones silíceas originadas por el producto protector contra la oxidación (arena o polvo de vidrio) que se aplicaba sobre el metal candente para evitar la oxidación debida a los sucesivos calentamientos necesarios para obtener la forma o la estructura deseadas. La inclusión inferior en la figura 1 parece responder a este tipo ya que su análisis (ver análisis $C$ en la tabla I) denuncia una naturaleza silícea casi pura que parece haber tenido poco que ver con la operación de reducción.

- Cascarilla propia del calentamiento. Las inclusiones halladas en la espada de Mondragón y en la falcata (Fig. 7 y Fig. 8 inferior derecha) ponen de manifiesto dos técnicas diferentes para obtener una espada: la primera, sigue la descripción de Jovellanos en sus memorias íntimas ${ }^{[6]}$; es decir, consiste en alternar y forjar juntos cuadradillos de hierro y acero, mientras que la falcata supone la obtención de un hojaldre de hierro-acero mediante sucesivas operaciones de doblado y forjado, (Fig. 6). En ambos casos, las inclusiones están compuestas de óxido de hierro con ausencia de otros elementos reveladores de su presencia en operaciones anteriores (ver análisis D y F en tabla I). Su aspecto al microscopio denuncia una naturaleza quebradiza.

\subsection{La calidad}

Según lo dicho hasta ahora, se puede considerar el proceso siderúrgico "antiguo" como la serie de operaciones que se llevaban a cabo con objeto de mejorar las propiedades de la masa de hierro reducida y adaptarla al uso previsto en cuanto a forma y eficacia. Uno de los casos más extremos es la fabricación de espadas que, aparte de la apariencia estética, debían ostentar unas características muy peculiares en cuanto a dureza para asegurar 


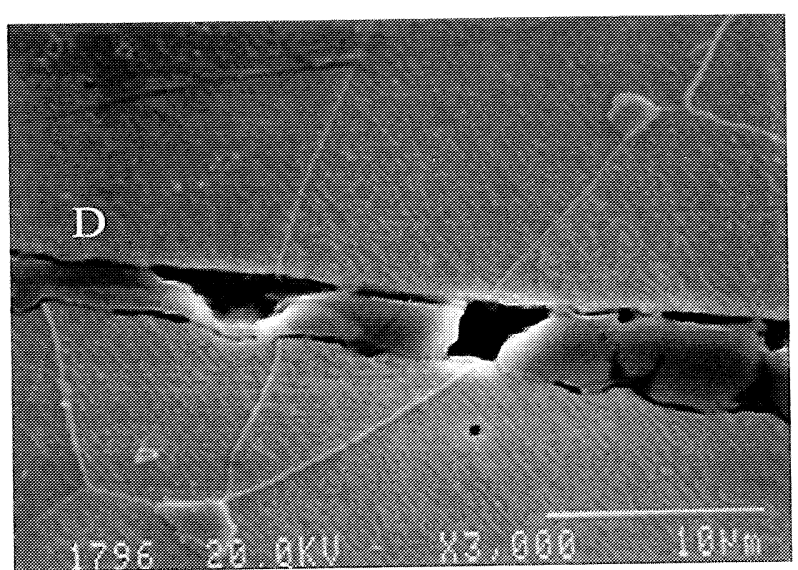

Figura 7. Inclusiones en falcata (MEB).

Figure 7. Inclusions in "falcata" (SEM).

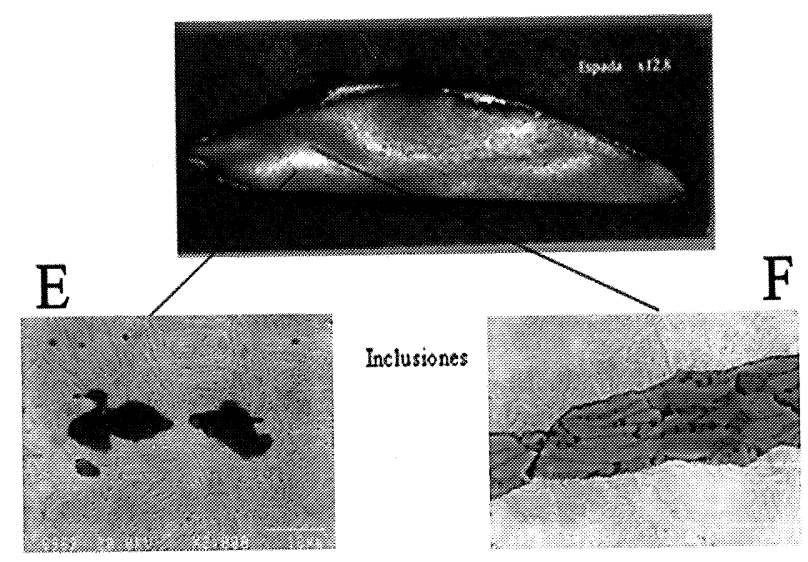

Figura 8. Inclusiones espada Mondragón.

Figure 8. Inclusions on "Mondragón" sword.

el corte, tenacidad para resistir los golpes propios de un duelo y capacidad de ser reparada y adquirir "temple" una y otra vez. No menos solicitadas están la mayoría de las herramientas agrícolas.

El amasado posterior a la reducción pretendía eliminar en lo posible las escorias ocluidas incrementando así la compacidad a favor de las características aludidas. Según esto, será posible establecer un criterio básico de evaluación de la calidad de un útil estudiando el tamaño y la distribución de las inclusiones. Según este criterio, podremos afirmar que un material cuya estructura presente el aspecto de la micrografía inferior derecha en la figura 5, correspondiente al mango de yunque estudiado, es de muy baja calidad ya que, al parecer, el trabajo de forja que ha sufrido es el indispensable para darle forma. Sin embargo, la falcata ibérica de la figura 9 presenta una cantidad

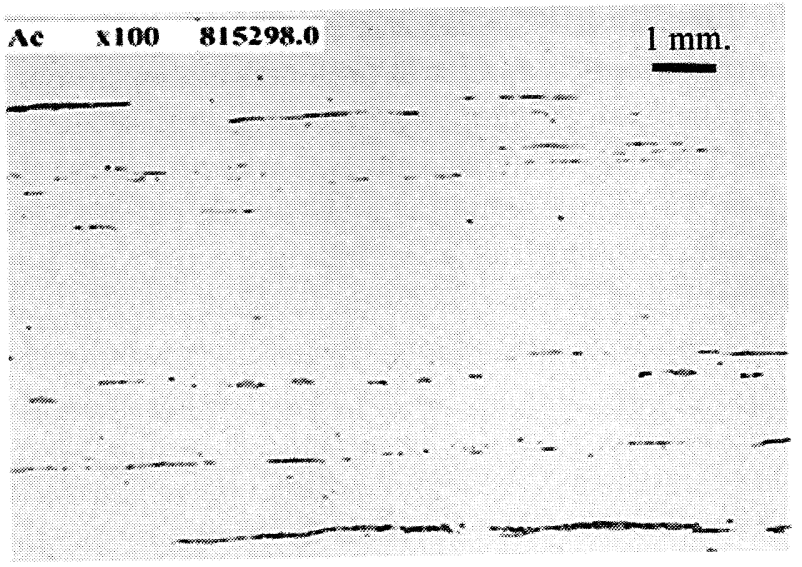

Figura 9. Inclusiones en falcata.

Figure 9. Inclusions in falcata.

moderada de inclusiones de espesor y longitud que responden a las sucesivas operaciones de doblado y forja necesarias para obtener el "hojaldre" que la constituye, por lo que podrá ser considerada como una herramienta de calidad para aquella época.

El carbono, como elemento clave para obtener mayor dureza asociado al hierro, no es conocido y controlado hasta bien entrado el siglo XIX; sin embargo, conocemos el uso consciente de materiales con mayor o menor contenido de carbono para obtener un resultado más favorable; éste, es el caso de la falcata en que se alternan capas de hierro y "acero" hasta un total de 32, en un espesor máximo de 3,5 mm.

De especial interés es el yunque elaborado según la técnica medieval de "calzar" el hierro, (Fig. 6) y que está compuesto de un soporte de hierro reducido según el proceso más tradicional, al que se ha soldado por calda un inserto de acero de la más alta calidad, ya que presenta una estructura prácticamente ausente de inclusiones (ver micrografía superior e inferior izquierda en la figura 5). Esta circunstancia se puede, quizá, explicar interpretando que se está ante un espécimen del famoso acero de Mondragón que, según nuestras referencias, pasaba por dos operaciones de licuefacción antes de ser considerado apto para el uso.

\subsection{La procedencia}

Los procedimientos analíticos, en especial la microsonda, permiten descubrir parentescos entre las inclusiones y los restos arqueológicos de operaciones de reducción o forja y aún del mismo mineral de origen ${ }^{[7]}$. 
En el caso de la falcata, se encuentra afinidad entre mineral y escoria al presentar ambos un porcentaje análogo de manganeso (ver análisis A y B en tabla I), elemento que posee la propiedad de pasar casi en su totalidad a la escoria; por otro lado, el relativamente alto contenido en calcio nos llevaría a relacionarla con un escorial con esta característica, lo que, según nos cabe saber, no debía ser muy frecuente en la época (siglo III a. de C.).

También habrá de considerarse la posible afinidad en cuanto al mineral de origen entre la espada y el yunque, ya que el análisis de las inclusiones de reducción ostenta análoga proporción de manganeso (ver análisis E y G de la tabla I), lo que puede ser relacionado con la proximidad geográfica de ambos lugares a las famosas minas del monte Udala cuyo mineral arroja una proporción significativa de este elemento (algo superior al 5\% en peso) en las pocas muestras que nos ha cabido analizar.

\section{CONCLUSIONES}

- El trabajo muestra las posibilidades para la determinación de importantes datos respecto al proceso de fabricación, a la calidad del objeto y al origen de las piezas arqueológicas mediante un estudio y análisis apropiado de las inclusiones presentes en dichas piezas.

- Un estudio más completo de la estructura y demás características de las piezas, permitiría de- terminar en mayor profundidad estos datos y otros nuevos los cuales, en contraste con datos históricos, permitirán aportar un mayor conocimiento desde los procesos utilizados por nuestros antepasados hasta los flujos migratorios de las piezas y por tanto de las personas que los utilizaban.

- Cabe destacar el hecho de que estos estudios han sido realizados mediante técnicas mínimamente invasivas, de tal forma que el valor intrínseco de las piezas analizadas se mantiene inalterado.

\section{REFERENCIAS}

[1] G. Rosado, El Acero de Mondragón; I Jornadas de Materialografía; San Sebastián, 2000.

[2] R.F. TyleCotTE; The Prehistory of Metallurgy; The Institute of Metals; London, 1995.

[3] C. DOMERQUE; Mélanges; Ed. Mergoil, Montagnat, 2000.

[4] V. Serneels ; Du Minerai à l'objet; Norberg Conference ; Ed.J.K. Stockholm, 1995.

[5] J.L. Mohen. Les Ciences du Patrimoine; Ed. Odile Jacob, Paris, 1995.

[6] G.M. Jovellanos; Diarios (Memorias Íntimas). Instituto Jovellanos, Gijón, 1914.

[7] G. RosADO; Caracterización de Útiles y Armas; II Jornadas de Materialografía; San Sebastián, 2002. 